\title{
Outage Probability of a Multi-User Cooperation Protocol in an Asychronous CDMA Cellular Uplink
}

\author{
Kanchan G. Vardhe, Daryl Reynolds and Matthew C. Valenti \\ Lane Dept. of Comp. Sci. and Elect. Eng. \\ West Virginia University \\ Morgantown, WV 26506-6109 \\ Email: kvardhe@mix.wvu.edu, Daryl.Reynolds@mail.wvu.edu, Matthew.Valenti@mail.wvu.edu
}

\begin{abstract}
In a recent paper, we proposed a multiuser space-time coded cooperative diversity protocol that operates in an asynchronous code-division multiple-access (CDMA) uplink under non-orthogonal channel assignment. The diversity combining of the relayed information was considered at the base station and the informationoutage probability performance was investigated in a highSNR regime. The goal of this paper is to extend those results and compare the performance of the proposed multi-user sharing protocol under diversity combining and code combining of the relayed transmissions at the base station and to examine the impact of using practical modulation techniques on the information-outage probability performance of the proposed multi-user cooperation protocol. We see that the performance loss due to modulation constraints and the use of diversity combining instead of code combining is relatively small.
\end{abstract}

\section{INTRODUCTION}

It is well known that multiple-input multiple-output (MIMO) communications have advantages of improving link reliability, and the capacity of wireless systems. However, the use of multiple antennas to achieve transmit diversity in the cellular uplink is impractical due to the size constraints of the mobile units. A potential solution is then to employ user cooperative diversity techniques whereby mobile users share their physical resources to create a virtual antenna array and hence achieve transmit diversity gain to combat fading.

The use of cooperative diversity in a cellular uplink was first popularized by Sendonaris et al. [1]. In [1], the authors develop a full-duplex, two-user sharing protocol for the code-division multiple-access (CDMA) using orthogonal spreading codes. However, the assumption of orthogonal spreading codes limits flexibility of the scheme. Orthogonality between spreading codes may also get destroyed due to asynchronous channels. In [2], the authors develop space-time coded decodeand-forward (DF) protocols for combating multipath fading in wireless networks and present informationoutage probability analysis of these protocols under repetition coding (diversity combining) in the high SNR regime. The medium access control protocol suggested in [2], [3], allocates orthogonal (frequency) channels to the transmitting terminals and also assumes block and symbol synchronization. The authors in [4] design linear multi-user detectors for the synchronous cooperative CDMA uplink using non-orthogonal spreading codes and analyze the performance of various detection strategies under repetition based full-duplex relaying schemes. The authors in [5], [6], [7] present various channel coding schemes for cooperative networks.

Most prior work on cooperative diversity builds upon the assumption of orthogonal channels to multiple users and synchronous communication between the signals transmitted from different cooperative terminals in the network. The issue of non-orthogonal channel allocation has been addressed in [9], [10]. The authors in [9] apply delay-diversity techniques to single source cooperative networks which do not require orthogonal channelization or symbol-level timing synchronization. In [10], the authors propose a cooperative transmission technique, where relay nodes act as active scatterers and simply retransmit the source's transmission under very loose synchronization constraints. Also, the previous information-theoretic analysis on user cooperation assumes Gaussian distribution of the input symbols, but practical systems must be constrained to use inputs selected from a finite signal set. The authors in [11] evaluate the impact of modulation constraints on the throughput of point-to-point hybrid-ARQ and suggest the extension to relaying protocols. Recently, a multiuser space-time coded cooperative diversity protocol that operates in an asynchronous CDMA cellular uplink under non-orthogonal channel assignment was proposed and diversity combining of the relayed information at the base station was considered [8]. The authors analyzed information-outage probability performance in three special cases such as underloaded, fully-loaded and overloaded CDMA uplink using the high-signal-tonoise ratio (SNR) approximations.

In this paper, we extend the results from [8]. In particular, we compare diversity combining (employing space-time coding) and code combining (employing incremental redundancy) at the base station using numerical results for the information-outage probability of the proposed DF sharing scheme in [8] under fully- 


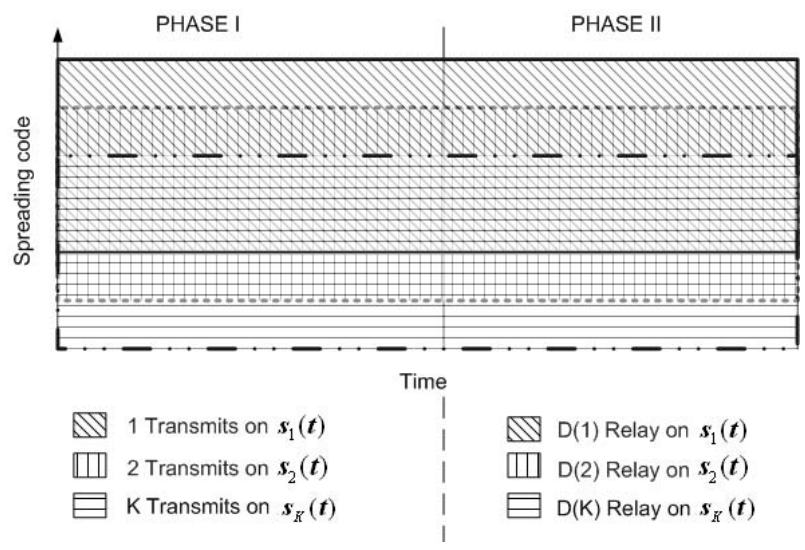

Fig. 1. Space-time coded medium-access control for the proposed cooperation scheme. Figure indicates example channel allocations across spreading codes and time for $K$ users. For user $k \in\{1,2, \ldots, K\}, \mathcal{D}(k)$ denotes the decoding set. The non-orthogonal (but linearly independent) spreading waveform of the $k$-th user is denoted by $s_{k}(t)$.

loaded CDMA uplink. It is well known that code combining is almost always better than the diversity combining in non-cooperative networks. This is because when code combining is used, individual channel mutual informations add while when using diversity combining, signal-to-noise ratios (SNRs) add. Interestingly, the results presented here indicate that in a multi-user cooperative diversity environment, diversity combining of the relayed information from multiple users is nearly as good as code combining because of the associated probabilities of a decoding set as will be explained in the sequel. We also examine the effect of using practical modulations on the outage probability performance.

The paper outline is as follows. Section II introduces a non-cooperative CDMA uplink model. Section III revisits and describes a proposed user cooperation protocol and received signal model from [8] for completeness. Section IV presents a multi-user cooperation protocol in a fully loaded CDMA cellular uplink under diversity combining while Section V considers code combining. The outage probability for the modulation constrained case is presented in Section VI. We provide the numerical results in Section VII and Section VIII concludes.

\section{Conventional CDMA}

In direct-sequence code-division multiple-access (CDMA) systems, each user is assigned an individual (orthogonal or non-orthogonal) signature waveform or a spreading code and signals from different users may overlap in both time and frequency. The received signal at the base station in a non-cooperative asynchronous CDMA uplink with $K$ active users is given by

$$
r(t)=\sum_{k=1}^{K} \sum_{i=0}^{B-1} x_{k}[i] \alpha_{k} s_{k}\left(t-i T_{s}-\tau_{k}\right)+n(t)
$$

where $B$ is the block length, $T_{s}$ is the symbol period, $n(\cdot)$ is the additive white Gaussian noise process, $x_{k}[i]$ is $k$-th user's transmitted symbol, $\alpha_{k}$ is the flat fading
Rayleigh channel coefficient for the channel between $k$-th user and the destination (base station), $s_{k}(t)=$ $\sum_{j=0}^{N-1} c_{k}[j] \psi\left(t-j T_{c}\right)$ is the spreading waveform of $k$ th user where $c_{k}[j] \in\left\{-\frac{1}{\sqrt{N}}, \frac{1}{\sqrt{N}}\right\}$ is the $j$-th element of user $k$ 's spreading code and $\psi(t)$ is a unit-energy transmit pulse shape waveform, $N$ being the processing gain.

\section{COOperation IN A CDMA UplinK}

\section{A. Protocol Design}

We analyze a user cooperation protocol wherein users transmit their own data and also serve as relays for other users in the system. This is in contrast with the typical relay networks where relays do not have data of their own. The relays can thus receive messages from multiple sources and upon successful decoding, can forward the superposition of multiple re-encoded and re-spread messages. The protocol description of the proposed multi-user cooperation scheme differs from [2] in medium-access control requirements and also in multiple access strategy. We consider a CDMA cellular uplink consisting of $K$ cooperating users. Each user is assigned a single spreading code. The spreading codes provide processing gain $N$ and are assumed non-orthogonal. Fig. 1 depicts channel and subchannel allotments for the proposed CDMA cooperative scheme. The channel representing a single spreading code spans two time-phases and when split into individual time phases corresponds to subchannels. The transmission between users and the base station is accomplished in two orthogonal time-phases. In the first phase, user $k \in$ $\{1,2, . . K\}$ transmits to the base station on its spreading code (i.e., in the appropriate subchannel). In the second phase, the users that can decode $k$-th user's transmission form a decoding set $\mathcal{D}(k)$ and serve as relays $(r)$. The relays then transmit to the base station asynchronously on source user's spreading sequence using a spacetime code or delay diversity technique which leads to diversity combining of the relayed transmissions at 


$$
\tilde{\boldsymbol{H}}=\left[\begin{array}{ccccccc}
\alpha_{1}^{*} \alpha_{1} \rho_{21}^{21} & \cdots & \alpha_{1}^{*} \alpha_{1} \rho_{21}^{K 1} & \cdots & \alpha_{1}^{*} \alpha_{K} \rho_{21}^{1 K} & \cdots & \alpha_{1}^{*} \alpha_{K} \rho_{21}^{K^{\prime} K} \\
\vdots & \vdots & \vdots & \vdots & \vdots & \vdots & \vdots \\
\alpha_{1}^{*} \alpha_{1} \rho_{K 1}^{21} & \cdots & \alpha_{1}^{*} \alpha_{1} \rho_{K 1}^{K 1} & \cdots & \alpha_{1}^{*} \alpha_{K} \rho_{K 1}^{1 K} & \cdots & \alpha_{1}^{*} \alpha_{K} \rho_{K 1}^{K^{\prime} K} \\
\vdots & \vdots & \vdots & \vdots & \vdots & \vdots & \vdots \\
\alpha_{K}^{*} \alpha_{1} \rho_{1 K}^{21} & \cdots & \alpha_{K}^{*} \alpha_{1} \rho_{1 K}^{K 1} & \cdots & \alpha_{K}^{*} \alpha_{K} \rho_{1 K}^{1 K} & \cdots & \alpha_{K}^{*} \alpha_{K} \rho_{1 K}^{K^{\prime} K} \\
\vdots & \vdots & \vdots & \vdots & \vdots & \vdots & \vdots \\
\alpha_{K}^{*} \alpha_{1} \rho_{K^{\prime} K}^{21} & \cdots & \alpha_{K}^{*} \alpha_{1} \rho_{K^{\prime} K}^{K 1} & \cdots & \alpha_{K}^{*} \alpha_{K} \rho_{K^{\prime} K}^{1 K} & \cdots & \alpha_{K}^{*} \alpha_{K} \rho_{K^{\prime} K}^{K^{\prime} K}
\end{array}\right], \boldsymbol{r}=\left[\begin{array}{c}
r_{2,1} \\
\vdots \\
r_{K, 1} \\
\vdots \\
r_{1, K} \\
\vdots \\
r_{K^{\prime}, K}
\end{array}\right], \boldsymbol{x}=\left[\begin{array}{c}
x_{2,1} \\
\vdots \\
x_{K, 1} \\
\vdots \\
x_{1, K} \\
\vdots \\
x_{K^{\prime}, K}
\end{array}\right]
$$

the base station or they may use different Gaussian codebooks (or incremental redundancy) which leads to code combining of the relayed transmissions at the destination. Thus for this cooperative diversity scheme, decoding relays for any particular source user transmit asynchronously over the same subchannel (i.e., they use the same spreading code). Note that since spreading codes are non-orthogonal, and we assume asynchronous communication between signals transmitted from cooperating users, we have non-orthogonality across the subchannels and also within a subchannel ${ }^{1}$.

\section{B. Received Signal Model}

The proposed sharing scheme operates in an asynchronous CDMA uplink in the presence of multipleaccess interference (MAI) and intersymbol interference (ISI). The specified use of decorrelating multiuser detection at the base station effectively transforms the resulting MAI and ISI channel into parallel interference-free scalar flat fading channels with increased background noise. Using this scalar channel model along with an appropriate signal-to-noise ratio parameterizations, the proposed scheme can be evaluated via outage probability, i.e., the probability that average mutual information (in bits/sec/Hz) falls below a given threshold. We now develop a signal model for the second phase of transmission but we note that the signal model for the first phase of transmission can be obtained in a similar manner. The received signal at the base station with $K$ cooperating users and $K^{\prime} \triangleq K-1$ potential relays is given by

$r(t)=\sum_{k=1}^{K} \sum_{l=1}^{K^{\prime}} \sum_{i=0}^{B-1} x_{l, k}[i] \alpha_{l} s_{k}\left(t-i T_{s}-\tau_{l}\right)+n(t)$

where $B, T_{s}, n(\cdot), \tau_{l}$, and $s_{k}(t)$ are as described under equation (1), $x_{l, k}[i]$ is $k$-th user's space-time coded symbol transmitted from $l$-th cooperating user with $\mathrm{E}\left\{x_{l, k}^{2}[i]\right\}=P, \alpha_{l}$ (or $\alpha_{l, d}$ ) is the flat fading Rayleigh channel coefficient for the channel between $l$ th user and the destination (base station) with variance $1 / \lambda_{l}$ (or $1 / \lambda_{l, d}$ ), and $\tau_{l}$ is the delay for the channel between $l$-th user and the destination. $\tau_{l}$ includes a random transmit delay for delay diversity. At the base

${ }^{1}$ But note that we still have time-phase orthogonality. station, the received signal is matched filtered with respect to the received waveform over the channel. By Cameron-Martin formula, this process generates sufficient statistics, $r_{k, l}[i]$ [12]. These are given by

$$
\begin{aligned}
r_{k, l}[i] & =\alpha_{l}^{*} \int_{-\infty}^{\infty} r(t) s_{k}\left(t-\tau_{l}-i T_{s}\right) d t \\
& =\sum_{k^{\prime}=1}^{K} \sum_{l^{\prime}=1}^{K^{\prime}} \sum_{i^{\prime}=0}^{B-1} x_{k^{\prime}, l^{\prime}}\left[i^{\prime}\right] \alpha_{l}^{*} \alpha_{l^{\prime}} \rho_{k l}^{k^{\prime} l^{\prime}}
\end{aligned}
$$

where $\rho_{k l}^{k^{\prime} l^{\prime}} \triangleq \int_{-\infty}^{\infty} s_{k}\left(t-\tau_{l}-i T_{s}\right) s_{k^{\prime}}\left(t-\tau_{l^{\prime}}-i^{\prime} T_{s}\right) d t$ is the cross-correlation between delayed spreading waveforms. Stacking all match filtered outputs, we get $\boldsymbol{r}=$ $\tilde{\boldsymbol{H}} \boldsymbol{x}+\boldsymbol{n}$ where $\boldsymbol{n} \sim \mathcal{N}_{c}\left(\mathbf{0}, N_{0} \tilde{\boldsymbol{H}}\right)$. This can further be expressed as

$$
r=\underbrace{A R A^{\mathrm{H}}}_{\tilde{H}} x+n
$$

where $\boldsymbol{A}$ is a diagonal matrix and is a function of only channel gains $\alpha_{i}$ 's, $\boldsymbol{R}$ is a function of cross-correlations between delayed signature waveforms. Applying the decorrelating detector [13] to the discrete-time received vector $\boldsymbol{r}$, we get $\boldsymbol{y}=(\boldsymbol{A R})^{-1} \boldsymbol{r}+\boldsymbol{v}$ where $\boldsymbol{v} \sim$ $\mathcal{N}_{c}\left(\mathbf{0}, N_{0} \boldsymbol{R}^{-1}\right)$. Thus we get a parallel flat fading scalar channel model similar to [2],

$$
y_{i} \triangleq[\boldsymbol{y}]_{i}=\alpha_{i} x_{i}+v_{i},
$$

but with enhanced noise distributed as $v_{i} \sim$ $\mathcal{N}_{c}\left(0, N_{0}[\boldsymbol{R}]_{i, i}^{-1}\right)$.

\section{PERformance UNDER DIVERSity COMBINING}

In this section, we study the performance of spacetime coded cooperative diversity protocol under diversity combining. In this type of cooperation, all the relays in the decoding set of a particular user transmit on the same subchannel using a space-time code or even using a delay diversity technique. The performance measure is information-outage probability that the average mutual information $(I)$ between user $k$ and the base station falls below a fixed spectral efficiency $R$ and is a lower bound on the codeword error rate of practically coded systems operating at the same spectral efficiency $R$. Since the decoding set for user $k, \mathcal{D}(k)$, is a random entity, the 
outage probability of the channel between user $k$ and the base station is given by

$$
\operatorname{Pr}[I<R]=\sum_{\mathcal{D}(k)} \operatorname{Pr}[\mathcal{D}(k)] \operatorname{Pr}[I<R \mid \mathcal{D}(k)] .
$$

We consider a fully loaded CDMA configuration in which $K=N$. Each user is assigned a single spreading code. Since each user sends its own data on its spreading code in the first time phase and also sends other user's data on that user's spreading code in the second phase, each user effectively uses up to all the spreading codes. Thus each cooperating user utilizes $1 / 2$ of available degrees of freedom in the channel. The $1 / 2$ factor is due to time-phase orthogonality and appears in front of the $\log$ terms. The normalized (by the degrees of freedom utilized by each cooperating user) discrete time power constraint is $2 P / K$ as described in [8]. Conditioned on the decoding set $\mathcal{D}(k)$, the mutual information between $k$-th user and destination can be shown to be

$$
\begin{gathered}
I_{\mathrm{f}-\mathrm{CDMA}}=\frac{1}{2} \log \left(1+\frac{2 \mathrm{SNR}}{K} \frac{\left|\alpha_{k, d}\right|^{2}}{\left[\boldsymbol{R}^{-1}\right]_{1,1}}\right) \\
+\frac{1}{2} \log \left(1+\frac{2 \mathrm{SNR}}{K} \sum_{r \in \mathcal{D}(k)} \frac{\left|\alpha_{r, d}\right|^{2}}{\left[\boldsymbol{R}^{-1}\right]_{r, r}}\right) .
\end{gathered}
$$

where SNR $\triangleq \frac{P}{N_{0}}$. The mutual information in (9) is the sum of the mutual informations for two parallel channels, one from the source to the destination and other from the set of decoding relays to the destination. Note that since we consider the relayed transmissions using a space-time code or a delay diversity technique, which yields to diversity combining at the base station, we have a log-sum expression for the second phase. The mutual information between the $k$-th user and the potential relay $r$ is given by

$$
I_{k, r}=\frac{1}{2} \log \left(1+\frac{2 \mathrm{SNR}}{K} \frac{\left|\alpha_{k, r}\right|^{2}}{\left[\boldsymbol{R}^{-1}\right]_{r, r}}\right) .
$$

The potential relay will be able to decode $k$-th user's message if the realized mutual information between user $k$ and the relay $r$ is greater than the fixed spectral efficiency $R$.

$$
\begin{aligned}
\operatorname{Pr}[r \in \mathcal{D}(k)] & =\operatorname{Pr}\left[I_{s, r}>R\right] \\
& =\exp \left[-\lambda_{k, r}\left[\boldsymbol{R}^{-1}\right]_{r, r} \frac{2^{2 R}-1}{2 \mathrm{SNR} / K}\right] .
\end{aligned}
$$

The probability of a decoding set is then given by

$$
\begin{aligned}
\operatorname{Pr}[\mathcal{D}(k)] & =\prod_{r \in \mathcal{D}(k)} \exp \left[-\lambda_{k, r}\left[\boldsymbol{R}^{-1}\right]_{r, r} \frac{2^{2 R}-1}{2 \mathrm{SNR} / K}\right] \\
& \times \prod_{r \notin \mathcal{D}(k)} 1-\exp \left[-\lambda_{k, r}\left[\boldsymbol{R}^{-1}\right]_{r, r} \frac{2^{2 R}-1}{2 \mathrm{SNR} / K}\right] .(13)
\end{aligned}
$$

Using (8), (9), and (13), we evaluate the outage probability performance of the above mentioned protocol numerically.

\section{PERFORMANCE UNDER CODE COMBINING}

The use of decorrelating multiuser detection as discussed in Section III-B allows us to form interferencefree scalar flat-fading parallel channels with increased background noise. Thus instead of repeating the same information in the form of a space-time code or delay diversity, the relays could as well employ different Gaussian codebooks and transmit relayed information toward destination. Therefore different part of the codeword gets transmitted from each cooperating user. This results in a code combining of the relayed transmissions at the base station. Under code combining, each subchannel in the second phase behaves like a set of $|\mathcal{D}(k)|$ parallel Gaussian channels. The mutual information under code combining and fully loaded CDMA system configuration, conditioned on a decoding set can be given by

$$
\begin{aligned}
& I_{\mathrm{f}-\mathrm{CDMA}}=\frac{1}{2} \log \left(1+\frac{2 \mathrm{SNR}}{K} \frac{\left|\alpha_{k, d}\right|^{2}}{\left[\boldsymbol{R}^{-1}\right]_{1,1}}\right) \\
& +\sum_{r \in \mathcal{D}(k)} \frac{1}{2} \log \left(1+\frac{2 \mathrm{SNR}}{K} \frac{\left|\alpha_{r, d}\right|^{2}}{\left[\boldsymbol{R}^{-1}\right]_{r, r}}\right) .
\end{aligned}
$$

The mutual information in (14) is larger than that in (9) for same $|D(k)|$ due to Jensen's inequality. Note that the existing cooperative diversity schemes employing code combining at the destination require the existence of parallel channels which is achieved through orthogonal channel allocation. But in our protocol, though the users have been allocated non-orthogonal spreading codes and relayed transmissions occur asynchronously in the same subchannel for each user, the decorrelating multiuser detector allows us to create virtual parallel channels without bandwidth penalty though there is a SNR penalty. The closed form expression for the outage probability under code combining is not tractable for arbitrary number of cooperating users. Hence we numerically evaluate the outage probability performance of the proposed protocol under code combining and compare it with the numerically evaluated performance of the proposed protocol under diversity combining (9). The final expression for the outage probability is obtained by substituting (14) and (13) in (8).

\section{PERFORMANCE UNDER MODUlation CONSTRAINTS}

In the earlier sections, we provided informationtheoretic analysis of multi-user cooperative diversity scheme using Gaussian distributed inputs. The assumption of Gaussian inputs is justifiable if we are dealing with large signalling constellations. However, the information-theoretic results need to be extended so as to take into account the effect of practical modulation constraints. In this section, we compute the mutual information under the constraint of uniform input probabilities considering diversity combining at the destination. To find the expression for mutual information 
under modulation constraints with the earlier mentioned system parameters, we model the received signal at the destination during two time-phases as follows. In the first phase, user $k$ transmits toward the base station. The received signal at the base station during first phase after decorrelating multiuser detection can be written as

$$
y_{1}=\frac{\alpha_{k, d}}{\sqrt{[\boldsymbol{R}]_{1,1}^{-1}}} x+n
$$

where $n \sim \mathcal{N}_{c}\left(0, N_{0}\right), x$ is a modulated symbol drawn from the uniform probability distribution with $\mathrm{E}\{x\}^{2}=$ $2 P / K$. The received signal model pointed out here is very similar to scalar channel model obtained in (7) except the scaled factor of $1 / \sqrt{[\boldsymbol{R}]_{r, r}^{-1}}$. We note that doing this does not change the received SNR (and yields exactly the same mutual information expression given in (9) assuming Gaussian distribution of input symbols) but allows us to separate out the effect of SNR from the interference term while plotting the outage probability performance. The mutual information under modulation constraints between $k$-th user and the base station during phase I conditioned on a decoding set $\mathcal{D}(k)$ is [15]

$$
I_{1}=\frac{1}{2}\left(m-\mathbf{E}_{x, y_{1}}\left[\log \frac{\sum_{z \in \chi} p\left(y_{1} \mid z\right)}{p\left(y_{1} \mid x\right)}\right]\right) .
$$

where $m=\log _{2} M, \mathrm{M}$ being the signal constellation size, $\chi$ denotes the signal set, and $p(y / x)$ is the transition probability density function between input $x$ and the output $y$ as defined in [15]. The factor $1 / 2$ outside the $\log$ term is due to the fraction of degrees of freedom utilized by a cooperating terminal in fully loaded CDMA system scenario as detailed in Section IV. Similarly the received signal at the base station during second phase under modulation constraints due to retransmissions from $K^{\prime}$ relays can be modeled as

$$
\boldsymbol{y}=\left[\begin{array}{c}
\alpha_{2, d} / \sqrt{[\boldsymbol{R}]_{2,2}^{-1}} \\
\vdots \\
\alpha_{r, d} / \sqrt{[\boldsymbol{R}]_{r, r}^{-1}} \\
\vdots \\
\alpha_{K^{\prime}, d} / \sqrt{[\boldsymbol{R}]_{K^{\prime}, K^{\prime}}^{-1}}
\end{array}\right] x+\boldsymbol{n} .
$$

Again, the expression for the mutual information under uniform input probability conditioned on a decoding set $\mathcal{D}(k)$ is given by

$$
I_{2}=\frac{1}{2}\left(m-\mathbf{E}_{x, \boldsymbol{y}}\left[\log \frac{\sum_{z \in \chi} p(\boldsymbol{y} \mid z)}{p(\boldsymbol{y} \mid x)}\right]\right) .
$$

The overall mutual information conditioned on a decoding set between $k$-th user and the base station is then

$$
I_{m}=I_{1}+I_{2} \text {. }
$$

The mutual information between $k$-th user and a relay can be formed in a similar fashion which then can be used to find a probability of a decoding set. We plot the outage probability performance through Monte-Carlo simulation using the total probability law in (8).

\section{RESULTS}

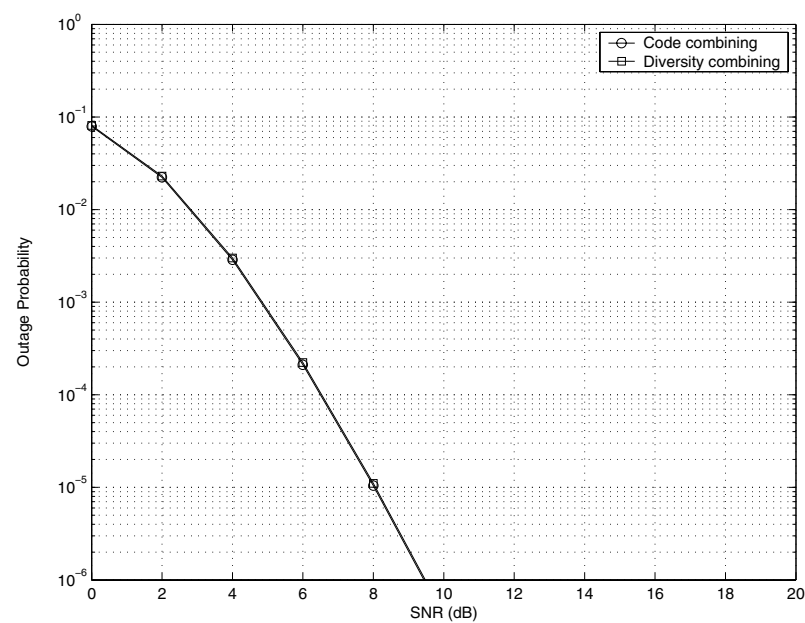

Fig. 2. Conditional outage probability performance comparison of diversity combining and code combining schemes for fully loaded CDMA system configuration with $K=N=8$. The outage probability is conditioned on $\boldsymbol{R}$. The threshold spectral efficiency is $R=1 \mathrm{bit} / \mathrm{sec} / \mathrm{Hz}$. Code combining is $0.01 \mathrm{~dB}$ better than the diversity combining and so the plots are almost indistinguishable.

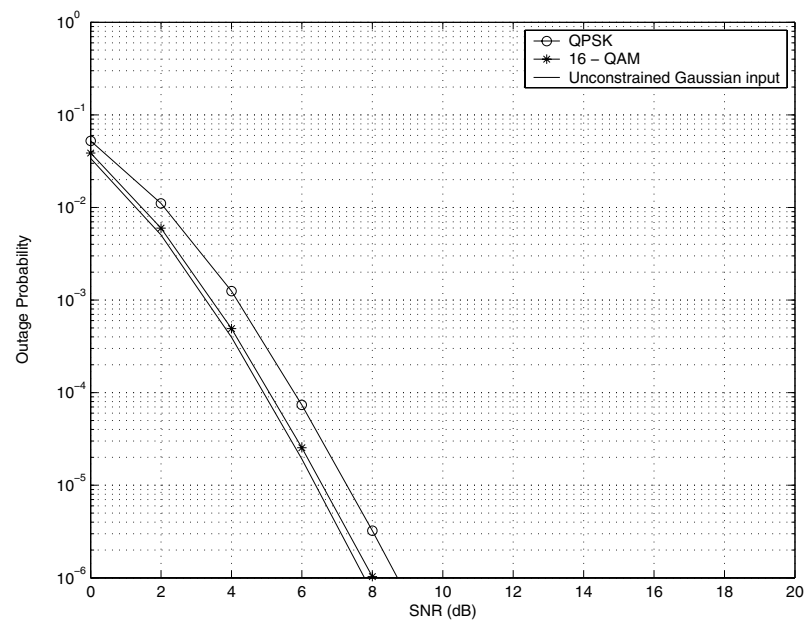

Fig. 3. Conditional outage probability performance comparison of fully loaded CDMA system configuration $(K=N=8)$ under the constraint of uniform input probability (QPSK and 16-QAM modulation) and unconstrained Gaussian input. The outage probability is conditioned on $\boldsymbol{R}$. The threshold spectral efficiency is $R=0.8$ bits/sec/Hz.

In all the figures, $N$ denotes the processing gain, and $K$ denotes the number of cooperating users. We consider a fully loaded CDMA uplink with $K=N=$ 8. The spreading codes are random and the delays are assumed to be uniformly distributed between 0 and $T_{s}, T_{s}$ being normalized to 1 . Fig. 2 indicates the conditional outage probability performance comparison between diversity combining and code combining 
reception schemes. As mentioned earlier, we present numerical results instead of high-SNR approximation. The outage probability is conditioned on $\boldsymbol{R}$ and hence is plotted for one realization of $\boldsymbol{R}$ for simplicity. We plot all the conditional outage probability curves using the same realization of $\boldsymbol{R}$. For other realizations of $\boldsymbol{R}$ also, the performance comparison remains the same. We recall that $\boldsymbol{R}$ is a function of cross-correlations between delayed spreading waveforms and does not involve channel gains. The results are plotted for $R$ $=1 \mathrm{bit} / \mathrm{sec} / \mathrm{Hz}$. It is well known that code combining is always better than the diversity combining in non-cooperative networks due to the consequence of Jensen's inequality. However, it can be seen from the figure that in a cooperative diversity scenario, under the system parameters mentioned in this paper, diversity combining is nearly as good as code combining. Specifically, code combining is $0.01 \mathrm{~dB}$ better than the diversity combining and this difference is not visible from the figure. This is because decoding set is a random variable. All potential relays in the system do not necessarily decode the source user's transmission simultaneously. For the SNRs of interest and fewer number of simultaneously active users in the system, the probability of having large number of relays in the decoding set is very small and therefore, considering the expansion of (8) in the increasing order of $|D(s)|$, only first few terms in the expression (8) dominate the system performance. Since the first few terms in diversity combining and the code combining are very similar, code combining does not offer performance gains (in terms of information-outage probability) over diversity combining. The conclusions might be different if we consider very high SNR regions and a large pool of users in the system. It was also observed that in a deterministic cooperative network, where $\operatorname{Pr}[D(k)]=1$ for some $D(k)$ (which is the case of a non-cooperative scenario with $|D(k)|$ parallel channels), code combining demonstrates significant performance gain in terms of information outage probability over diversity combining. Thus the probabilities of the decoding sets drastically affect the performance of a cooperative protocol under diversity and code combining schemes.

Fig. 3 compares the conditional information-outage probability performance under modulation constraints and also unconstrained Gaussian input distribution assuming diversity combining at the base station. The information-outage probability is conditioned on $\boldsymbol{R}$. We plot the curves for QPSK modulation and 16-QAM modulation against the threshold spectral efficiency $R=$ $0.8 \mathrm{bit} / \mathrm{sec} / \mathrm{Hz}$. It is seen that increasing the signal constellation size renders similar performance to Gaussian input distribution performance at lower rates.

\section{CONCLUSION}

In this correspondence, we compared diversity combining and code combining at the base station for the cooperative diversity that operates in an asynchronous CDMA uplink under non-orthogonal channel assignment. It is seen that in multi-user cooperation, diversity combining yields almost the same outage probability performance as code combining because not all users in the system act as relays all the time and hence the probabilities of the decoding sets turn out to be a prominent factor in deciding which combining scheme to use at the base station. We also evaluated the performance of multi-user cooperation protocol under the practical modulation techniques. It is observed that increasing the signal constellation size while keeping the target rate constant, we can approach the outage probability performance of a cooperation scheme that uses Gaussian inputs. Looking at all the results, we can argue that the performance loss incurred (with respect to their counterparts) by making the system design much simpler and more practical, for e.g., using diversity combining (instead of code combining), and a 16-symbol alphabet, is relatively small.

\section{REFERENCES}

[1] A. Sendonaris, E. Erkip, B. Aazang, "User cooperative diversity Part I: System description," IEEE Trans.Commun., vol. 51, no.1, pp. 1927-1938, Nov. 2003.

[2] J. N. Laneman and G.W. Wornell, "Distributed space-time coded protocols for exploiting cooperative diversity in wireless networks," IEEE Trans. Inform. Theory, vol. 49, pp. 2415-2525, Oct. 2003

[3] J. N. Laneman, D. Tse and G. Wornell, "Cooperative diversity in wireless networks: Efficient protocols and outage behavior," IEEE Trans. Inform. Theory, vol. 50, no. 12, pp. 3062-3080, Dec. 2004.

[4] L. Venturino, X. Wang, M. Lops "Multiuser detection for cooperative networks and performance analysis," IEEE Trans. Signal Proc., vol. 54, no. 9, pp. 3315-3329, Sept. 2006.

[5] T. Hunter and A. Nosratinia, "Coded cooperation under slow fading, fast fading and power control," in Asilomar Conferencce on Signals, Systems, and Computers, Nov. 2002.

[6] T. Hunter, S. Sanayei, A. Nosratinia, "Outage analysis of coded cooperation," IEEE Trans. Info. Theory, vol. 52, pp. 375-391, Feb.2006.

[7] A. Steafnov, E. Erkip, "Cooperative coding for wireless networks," in IEEE Conference on Mobile and Wireless Coтmunications Networks, Stockolm, Sweden, Sept. 2002.

[8] K. Vardhe and D. Reynolds, "The space-time coded cooperative diversity in an asynchronous cellular uplink," in Proc. IEEE Military Commun. Conference, (MILCOM), Washington DC, Oct. 2006.

[9] S. Wei, D. Goeckel, and M.C. Valenti, "Asynchronous cooperative diversity," IEEE Trans. Wireless Commun., vol. 5, April 2006.

[10] A. Scaglione and Y. W. Hong, "Opportunistic large arrays: Cooperative transmission in wireless multihop ad hoc networks to reach far distances," IEEE Trans. Signal Proc., vol. 51, pp. 2082-2092, Aug. 2003.

[11] T. Ghanim and M. Valenti, "The throughput of hybrid-ARQ in block fading under modulation constraints," in Proc. Conf. on Info. Sci. and Sys. (CISS), (Princeton, NJ), Mar. 2006.

[12] H. V. Poor, An Introduction to Signal Detection and Estimation, 2nd ed., Springer, 1994.

[13] S. Moshavi, "Multi-user detection for DS-CDMA communication," IEEE Commun. Magazine, vol. 34, no. 10, pp. 124-136, Oct. 1996

[14] V. Tarokh, H. Jafarkhami, and A. R. Calderbank, "Space-time block codes from orthogonal designs," IEEE Trans. Inform. Theory, vol. 45, no. 5, pp. 1456-1467, July 1999.

[15] G. Caire, G. Taricco, and E. Biglieri, "Bit-interleaved coded modulation," IEEE Trans. Inform. Theory, vol. 4, no. 3, pp. 927 946, May 1998. 